which is given in Table III, dates were first soaked in water until swollen and then ripened at interval of ten degrees from $40 \mathrm{deg}$. Cent. to $90 \mathrm{deg}$. Cent. TABLE III.-EFFECT OF DIFFERENT TEMPERATURES

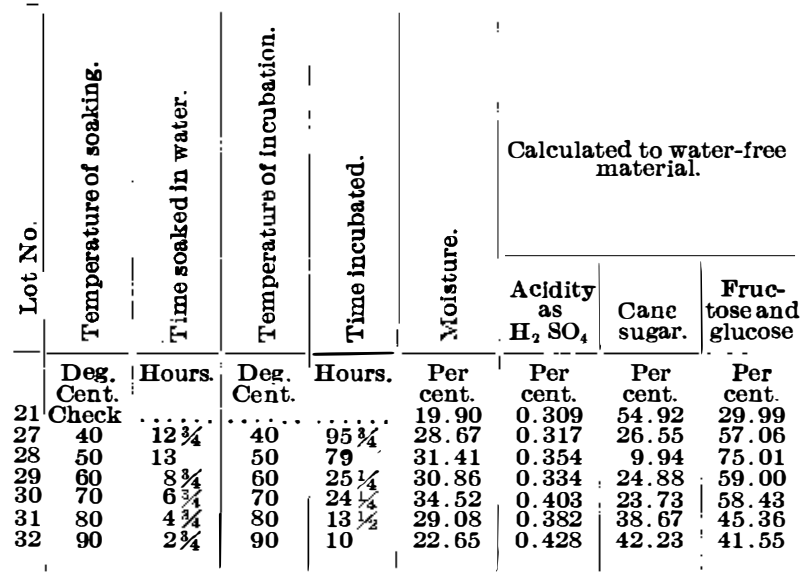

It has been found that attempts to conserve cane sugar by ripening the dates at or below $40 \mathrm{deg}$. Cent. result in prolonging the exposure necessary for the completion of the process and strongly increases the liability to sour. On the other hand, at temperatures above $55 \mathrm{deg}$. Cent. (55 to 65) although not more than half of the time necessary for ripening at $50 \mathrm{deg}$. Cent. is required, great care must be exercised in order to prevent the production of dark colored, sticky dates. Although there is more cane sugar present, dates ripened at these high temperatures do not have so pleasing a flavor as do those ripened with less heat.

Prof. Freeman holds that the inverting agents of the Deglet Noor date do not reach their greatest activity under the conditions surrounding the trees as grown up to the present time in Arizona. Such of these dates as naturally ripen in this climate, therefore, having very little of their sugar inverted. Their inverting agents require for their greatest activity, temperature distinctly higher than occur naturally during the ripening season in Arizona.

He claims that the ripening of a cane sugar date is a process separate and distinct from the inversion of the cane sugar present, and that conditions favorable for the rapid ripening of the Deglet Noor date may be produced artificially in an oven by regulating the degree of moisture and temperature. In this ripenin process the tissues of the date are softened and the tannin is precipitated, thus relieving the date of it astringency.

\title{
High Vacua: Their Production and Properties
}

\author{
The Radiometer as a Delicate Pressure Gage
}

\section{By IV. R. Whitney}

THE purpose of this article is to publish a iew observations made in the research laboratory on some phenomena in vacua, as they may possibly be of interest to others who are working in the field of evacuated apparatus.

The subject has a double interest because at first glance it seems difficult to see how there can be much ground for study, or material and phenomena for useful investigation in such an apparently confined volume of nothingness as the vacuum of an incandescent lamp. The many actual developments from this lack of material are interesting, and there is the added interest, due to the fact that there are to be found in these lamps examples of many of the phenomena of gaseous ions which are receiving so much study in our day.

It has long been known that the life of an incandescent lamp is greatly influenced by the quality of its vacuum. In general, one may say that the better the vacuum, a least up to a certain point and until recently, the longer the life of the lamp. In the early days of lamp making many schemes were devised to improve the vacua. This was then all the more necessary, as the mechanical methods of lamp-exhausting were very much inferior to present ones. The harmful effects of oxygen and water vapor, both of which reacted upon the filaments, were well known and many ways were devised for removing

What seems to be an especially interesting method of supplementing the vacuum pumps was the method of Malignani. This process, in its most perfect form, consisted in distilling into the bulb a small amount of some such substance as arsenic, iodine, or phosphorus. At the instant when one of these vapors was introduced, he passed a high current through the filament, the lamp being closed from the pump. This has long been the common commercial process for lamps which are to operate on voltages above 50 , and many millions of lamps have been exhausted by its aid.

In the case of incandescent lamps where the voltage is above 50 for a fair brilliancy of filament, a bluedischarge passes through the bulb and this blue quickly disappear when such vapors are introduced. The blue discharge seemed to be necessary for any considerable improvement of the vacuum, due to such vapors as phosphorus, and this led to the assumption that the gases still left in the lamp by the pump, were removed quickly during the blue glow and probably by the vapors which were at the same time being deposited on the glass. Although the facts were pretty well known, we performed experiments to study the phenomena. It was found that when the vacuum was measured by a McLeod gage, the sudden marked improvement of vacuum was easily proven. For example, in experiments carried on by $\mathrm{Mr}$. proven. For example, in experiments carried on by Mr. Willey, of the laboratory, when the pump had produced
a vacuum of 0.030 millimeter mercury and the cock a vacuum of 0.030 millimeter mercury and the cock
leading to it was closed, a trace of phosphorus was disleading to it was closed, a trace of phosphorus was dis-
tilled into the bulb while the filament was very hot and a blue glow had appeared; then about as quickly as th gage could be read the pressure had fallen to 0.002 or 0.001 millimeter.

* Paper presented to the American Institute of Electrical En-
ginoers and published in its Proceedings. Copyright, 1912, by
That this improvement of vacuum is quite commonly produced when vapors of any kind are caused to condense in the space is not new. It even takes place markedly when the filament is first heated to very high temperature without added vapor, and a blue glow also passes through the lamp at the time. In improving the vacuum this latter way, however, it is known that th filament is injured and apparently a part of its material has been vaporized. This process soon causes blackening of the bulb by carbon.

This vaporization of phosphorus into the lamp at the moment when the pump has done its work, has long bee the commercial method of finishing the exhaustion of incandescent lamps. The fact that even the carbon alone tended to the same end, though at the expense of the filament, being recognized, it became of interest to get a clearer view of this phenomenon.

In other words, if an incandescent lamp was burned while connected to a McLeod gage and the vacuum became poorer, the changes of vacuum might be measure by the gage from time to time. If, on the other hand, the vacuum improved so that the pressure tended to become less than the vapor pressure of mercury at th temperature of the gage, then the gage would not only not measure it, but the mercury vapor might even have affected the life of the lamp. When experiments wer made to determine the effect of the vapor of mercury at the low pressures corresponding to a micron, which is peculiar results were obtained and the lamps showed ver early blackening. It looked as though even the presence of this constant mercury pressure was fatal to the lamp. Therefore the discovery of a more practical vacuum gage was desirable.

Without committing ourselves to a theory, we can describe one or two interesting experiments with mercury vapor. It was first discovered that an incandescent lamp grew black very quickly when attached to mercury column which served as a gage to indicate evolution of gas. No such combination could be made to last over a few hours. The lamp blackened just as though it were in an imperfect vacuum. It is hard to see how the mercury can have any chemical effect upon the filament as oxygen or water have, and the effect is common to carbon and tungsten lamps. Many lamps were then made which were exhausted as perfect lamps are exhausted, except that a small quantity of mercury was left in the bulb. It was assumed that this would not interfere with the removal of air and moisture and might even assist by the washing effect of the mercury vapor, mechanically removing air. Lamps were also made in which a large quantity of mercury was placed, the lamp put into an oven above the boiling-point of mercury and the air washed out by the distilling mercury, no pump being used. When the mercury was nearly all removed the bulb was sealed off. This method, if no visible mercury is left in the bulb, is capable of giving good lamps, but where a visible drop of mercary was present the lamp would show a persistent blue glow and rapidly blacken, even below its normal voltage. In some cases it would are between the leads, exactly as in the case of poorly exhausted lamps.
It is further claimed that the same conditions of moisture and temperature used by Prof. Freeman in ripening Deglet Noor dates were also favorable for great activity of the inverting agents present in these dates. The artificially ripened product therefore differs from that naturally ripened in having nearly all of its sugar in the inverted form.

It is rather interesting to note how the application pe the methods steps in at every point of industry, and seems to be calculated in this particular instance to establish in our own country the come from the more tropical regions of the past has The case might be regarded as a peculiar type of adaptation; since the palm tree cannot adapt itself to the climates in which we live to the extent of ripening its fruit for our use, the ingenuity of modern man steps in and substitutes, as it were, an artificial climate during the last stages of the ripening process. We might say, the palm will not adapt itself to the climate, then we must adapt the climate to the palm."

The University of Arizona is to be congratulated on the work done by its agricultural experimental station in developing this new process of ripening dates by cubation.
Lamps were also made to which were attached tubes which carried small globules of mercury. When these side tubes were short and straight, such as one or two inches, and the lamp was exhausted as well as possible on the pump, the blackening of the bulb started at once when the entire glass was at ordinary temperature. If now, the side tube with its mercury was submerged in different cooling mixtures, the length of time for a given blackening was increased. 'For example, at room temperature the lamp would be blackened so as to correspond to 80 per cent of its original candle-power in a few minutes. When the side tube containing the mercury was at $0 \mathrm{deg}$. Cent. this time became two or three hours; and when cooled in a freezing mixture at $20 \mathrm{deg}$. Cent. the life to 80 per cent was over 75 hours. Such differences were also noted when the pressure of mercury vapor was controlled by using cadmium amalgams, the amalgams higher in mercury corresponding to the shorter lamp life.

It was interesting to note also that by lengthening the side tube containing the mercury, the rate was decreased at which this filament material was deposited in the bulb. In a set of experiments at ordinary temperatures the length of the side tube was increased and it was wound as a spiral. This reduced the rate at which the mercury could reach the bulb proper. In this case, instead of there being a continuous faint blue glow; as there is when the mercury is in a short side tube, the blue glow appeared and disappeared continuously and regularly. It looked as though there was a certain pressure of the vapor necessary before the blue discharge could occur and that when this was reached, the sudden discharge produced the blue and cleared the vacuum to a much lower pressure. Then mercury vapor distilled anew from the long side tube until again the necessary pressure was reached, when the process of clean-up repeated itself. If the lamp bulb was kept very hot, the blackening was reduced and even done away with altogether, as though the deposition of the mercury vapor upon the glass by the discharge signalized by the blue glow could not take place. If, however, water vapor was present, even in very small amount, the blackening would take place very rapidly, even in the absence of mercury. In other words, most ordinarily, exhausted lamps will blacken relatively quickly if allowed to burn in a heated oven. In general, the hotter the oven the more rapid the blackening, but this process is largely, if not entirely due to imperfect exhaustion. All glass contains water which can be removed but slowly, even at relatively high temperatures. It has even been found that indefinite heating of a lamp connected to the vacuum pump is not capable of removal of all of this water. If exhaustion be carried on for a very long time at room temperature then merely raising the temperature will cause liberation of more gas and after heating and exhausting to an equilibrium condition at say $200 \mathrm{deg}$. Cent. more water will be produced on heating to $300 \mathrm{deg}$. Cent. and still more at 400 degrees. For this reason it is customary to exhaust at as high a temperature as possible. At 400 deg. Cent. the ordinary glass has reached its limit, owing to the proximity of the softening point. For this reason we have had to exhaust the oven in which the lamps were 
being exhausted, in order to study the water evolution at higher temperatures.

For a long time it was impossible to follow the change of vacuum in a lamp, because the pressure could very evidently not be reduced-below the vapor pressure of the mercury of the McLeod gage attached to the lamp. It has long been known also, that the current passed through the space of the bulb to a considerable extent, and that this varied with the degree of vacuum, etc. A very complete discussion and description of experiments on this so-called Edison effect are given by Prof. Fleming, in Vol. 42 of the Philosophical Magazirue. In general, the conclusions are that in the vacuum of an incandescent lamp a current can flow across the space from the negative end of the filament to any conductor in the bulb, if this be so located that particles passing in straight line from the negative leg may come into contact with this conductor. This latter was usually a small platinum plate, and to it, as anode, a continuous current flowed from the negative half, or end of the filament, except when it was intermittently shielded from direct emanations. The current often of several milliamperes was measured by a galvanometer connected between the metal plate and the positive terminal of the lamp. It is known that the materials of the filaments distill in straight lines and cast well defined shadows, because the mean free path at this pressure is greater than the diameter of the bulb, and it was natural to assume that this vaporizing carbon carried electric charges of negative sign. This assumption might seem to acquire the more rapid wasting away of the negative half of the filament than the positive, but no data are present to prove this. When condenser charges were superposed on this apparatus it was found that with the negative end connected with the negative side of the condenser, the condenser was at once discharged. When connected oppositely, it was not discharged. Current could pass as negative from the hot conductor or filament, but not from the
cold plate, nor could positive current pass from either the hot or cold terminal.

In connection with this work of Fleming's, we have studied the effect of the condition of vacuum on the current, and also found that the presence of gas is necessary for this negative discharge. In general, the higher the pressure of the gas when this does not exceed a few thousandths of a millimeter mercury, the greater is the current. This makes it seem improbable that the current is solely dependent on the passage of carbon or tungsten from the filament to the positive elestrode, though it leaves the possibility open that the current may be carried by gaseous ions produced by negatively charged material coming from the negatively charged filament.

By using alternating current and a metal electrode in a carbon lamp, Fleming obtained direct current on the circuit connecting the metal plate to either terminal of the lamp and the current was in the direction expected, the plate being negative to the terminal.

This outfit is thus obviously a rectifier, and it has been so used by Fleming for low currents.

The effect of a magnetic field on this gaseous conduction, just as in the case of metallic bismuth, is to increase the resistance.

It was finally found that the Crookes radiometer was a very sensitive gage for the changes of pressure in lamps, and by its use the changes could be followed in a qualitative manner, even throughout the life of the lamp, by having the radiometer sealed directly to the lamp. Dewar has shown the sensitiveness of this apparatus in the Proc. Roy. Soc., 1907, 531.

The radiometer consists of a glass bulb containing a perpendicular needle with a glass cap on which are four aluminium arms with a mica vane at the end of each. One face of each mica vane is coated with lamp-black. When the radiometer is evacuated to a high degree, the effect of radiant energy or light is to cause rotation, which is believed to be due to the fact that the molecules of gas take up energy from the blackened surface of the vanes more than from the plane surfaces, the black absorbing more of the incident energy and thus locally heating the gas molecules. These in the rarefied atmosphere largely give off this energy to the walls of the apparatus by direct impact, rather than to other moleapparatus by direct impact, rather than to other mole-
cules in the neighborhood of the vane. This results in a motion of the vane opposite to the motion of the gas molecules, because the unblackened face of the vane receives impact mostly from these cooler molecules.

The vacuum of the lamp as sealed from the pump approximates a few microns and is poor enough so that the radiometer rotates rapidly when exposed to light. By using a fairly constant light intensity and noting its effect upon a radiometer attached to a lamp which was lighted for intervals, it was seen that the radiometer gradually rotated slower and slower, and finally after a period of normal lamp brilliancy from 12 to 36 hours on tungsten lamps, it would stop rotating altogether. At this point it was found that a more sensitive measure of still higher vacua could be gained by starting in the dark by mechanical motion a rotation of the vanes of a definite speed, and noting the rate of speed decay on standing.
This was done by proper spinning of the whole apparatus in the hand and then getting the rate of decrease in this rotation of the vanes by counting definite revolutions after definite time intervals. Here the friction of the residual gases was merely overcoming the momentum mparted mechanically to the vanes at start. In this way even by comparing the minutes required for the radiometer to come to a stop, quite interesting information was obtained.

This method was not developed into a quantitative one, though it might have been. The radiometer method shows us, however, some interesting facts which are worth describing. Using the common type of radiometer often seen in jewelers' windows and sealing this directly to a lamp made from 110 volt circuit, the radiometer would rotate when in daylight. This showed the presence of some gas in the lamp and the MeLeod gage used at the time of sealing the lamp from the pump showed approximately 2 microns. If, now, the radiometer and the lamp were removed to a dark room and a rotating motion imparted to the vanes, they would proceed to rotate for about a minute, this time depending somewhat on the particular radiometer used, as well as on the condition of the vauum.

Since at the moment when the filament was at bright heat and the vacuum very poor (so poor, in fact, that if the pressure were maintained constant the lamp would blacken in a few minutes), the vacuum greatly improved, owing to some action occurring together with spattering of the filament, it seemed important to know more about the phenomena. Wo assume that the gas at first present goes to the walls of the lamp and is held there, possibly abscrbed by the small quantity of deposited filamentmaterial It is always possible to drive the greater part of it back into the vacuum space by warming the glass. It seemed perfectly possible that the process might be a cyclic one that is, the gas carrying to the walls of the bulb some of the filamont material and not of necessity remainin there, but possibly returning for a fresh supply. This continued process might account for the limited life of an incandescent lamp. It did not seem necessary to distillation from a carbon filament at the temperature of operation, though such a phenomenon could account for limited life of filament. It even seemed possible that the cyclic process, instead of being physical, might well be expressed as a chemical one, such as we know could exist, and might continue between the oxides of carbon and carbon itself. At the high temperature of the filament carbon monoxide would be expected but this, at a lower temperature, would form $\mathrm{CO}_{2}$ and free carbon, the latter being deposited upon the glass. The free $\mathrm{CO}^{\circ}$ coming into contact with the hot carbon filament would again form carbon monoxide, and thus the process b repeated continuously until the filament was burned through or the lamp became too black to be of use. It was therefore desirable to get a closer insight into the phenomena in the lamp at a few microns pressure.

Similar considerations can apply equally well to the case of tungsten filament lamps. In the case of osmium lamps this reasoning extended, explains the fact that the presence of a little oxygen in the lamp was necessary to prevent blackening of the bulb by the deposited metal. deposited carbon from the glass and deposit it upon the filament, if the temperature at the two points were suitable, but with this element it seems impracticable because the oxide formed is not easily reduced below the CO state, thus differing from osmium.

While the lamp to which a radiometer was attached was not lighted, practically no change in vacuum could be detected by the radiometer. Therefore there is no leak of air from without. 'If, however, the lamp wa lighted, the reverse was true. On allowing the lamp to be lighted for a few seconds only, and then cooling and testing in the dark room as before, the duration of rotation would usually be shorter, thus indicating a libera tion of gas into the bulb space by this short time of burning. If, however, the lamp were allowed to burn at a normal brilliancy for a longer time and the same vacuum test was made from time to time on the unlighted lamp in the dark room, it was found that the duration of the impressed rotation rapidly increased. In many cases rotation lasting 15 minutes would be produced by allowing the lamp to burn 24 hours. This phenomena of automatic vacuum improvement received the name of the "clean-up." While the clean-up, as measured in thi rough way, did not seem to take place in all lamps to th same degree. it was always present under ordinary conditions.

Other types of vacuum lamps improved their vacua on running. In fact, no lamp, unless we except the mersystem of lighting, which is a development of the Crookes tube, consumes the gas, and for that reason an automatic valve was invented which supplier air to the tube when the vacuum improves to a certain point. It is also an old story, in the case of the cathode and X-ray tubes. $\mathrm{X}$-ray tubes are usually made with a salt, such as potas- sium chlorate or potassium hydrate, mica, asbestos, etc., tube in use attains too high degree of evacuation and in this way gas is liberated into the bulb. This is in turn removed from the vacuous space by the operation of the tube. A natural question is raised at once: what becomes of these disappearing gases? In some cases they are probably forced or shot into the glass itself, for some of these old glasses bubble on being heated to the softening point. Possibly the greater part of the gas is absorbed by the exceedingly finely divided metal, which slowly deposits on the glass as it vaporizes from the elec-

If an incandescent lamp, the vacuum of which had been thus cleaned up, was allowed to stand unlighted at ordinary temperature, the vacuum, as indicated by the test, would gradually grow poorer, though it would seldom fall to the starting value. If the bulb were heated in an oven at $100 \mathrm{deg}$. Cent. for a few moments, it would also show a poor vacuum again on cooling. If such a spoiled vacuum lamp was again lighted, it would at once commence to recover its previous high vacuum. There was no indication of a fatigue evinced in lamps thus treated.

This explains the fact noticed in the case of most factory made incandescent lamps, that their life at constant voltage varies with the external temperature. It is as though the gas, which is at first present in the space of the sealed bulb and is then thrown upon the walls by the clean-up, is also continually driven off from the walls at a rate dependent upon the wall temperature, and the deposition is bound up with some loss of filament mar terial.

The experiments with the radiometers naturally led to attempts to develop more nearly quantitative apparatus which would still measure the low pressures of gas under consideration in incandescent lamps. Among the promising methods was that described by Pirani (Ber. Deut. Phys. Ges. 1906, 686). This was further developed by Dr. Hale of this laboratory, as described in the Transactions of the American Electrochemical Society, 1911.

This gage depends on the principle that energy loss from a heated wire in the vacuum to be measured depends on the pressure of the gas present. When the temperature of this wire is low (100 deg. Cent., for example), the losses by radiation are relatively small, so that the rate of loss at a constant temperature of wire would serve to determine the gas pressure. Con versely the temperature of this wire, if supplied with constant energy, from the battery, for example, would be higher the lower the gas pressure. This latter scheme was adopted by $\mathrm{Dr}$. Hale, and the gage consisted in a glass bulb sealed directly to the lamp to be measured and containing about two feet of fine platinum wire. This was heated by a current of constant watts and the temperature of the wire was given by knowing its temperature resistance coefficient

The measurement involved extrapolation toward perfect vacua from comparative measurements made with the new gage and the McLeod gage at pressures high enough so that the latter is reliable. Hale concluded that he could measure differences of pressure of one hundred thousandth of a millimeter of mercury, or a hundredth part the vapor pressure of mercury at zero centigrade. Here was a gage which, unlike the McLeod, exerted no appreciable vapor pressure of its own and therefore could serve to indicate the existing pressure at any period of the life of the lamp. It could disclose the fact if gases were produced within the burning lamp, if the seals or glass leaked at all, and how the clean-up actually changed the vacuum as indicated by the radiometer experiments. In using this gage new and interesting phenomena were disclosed, which must be further studied. In certain cases vacua a little more than perfect are indicated by this gage, and this suggests possible dissociation of the very attenuated gas. Barring this fact, it seems as though the clean-up effect proceeds to as tively.

Transfer Paper.-Distribute 250 parts of pure starch in a small quantity of cold water and add 1,500 parts of boiling water, slowly and constantly stirring. To this starch-water add, stirring slowly, a mixture of 10 parts of neutral chrome yellow and 4 parts of gum arabic, dissolved together in water. To the whole 500 parts of glycerine are added, and to prevent the formation of a crust, the whole is kept in motion until completely cooled. To free it from undissolved or other solid particles, it must be carefully pressed through fine bolting cloth and is then ready for application to the paper. This is effected by means of soft brushes, particular care being taken to insure even distribution. The finished sheets are dried by suspension in the air. The paper remains moist and ean, therefore, be kept in an unrolled condition. This makes the moistening of the stone or the plate unnecessary and the transfer retains the same size as the negative. 